\title{
Validación de protocolo para transversalizar la perspectiva de género en las prácticas de diagnóstico de la carrera de psicología
}

Gloria M. Mora-Guerrero*; Andrés S. MacAdoo-Espinoza; Jorge C. Troncoso-Arcos; y Loreto A. Riquelme-Bravo Universidad Católica de Temuco, Temuco - Chile. (correo-e: gmora@uct.cl; amacadoo2014@alu.uct.cl; jtroncoso@uct.cl; Iriquelme@uct.cl)

* Autor a quien debe ser dirigida la correspondencia.

Recibido Dic. 10, 2020; Aceptado Feb. 9, 2021; Versión final Abr. 24, 2021, Publicado Ago. 2021

\section{Resumen}

Este estudio describe el proceso de construcción y validación de un protocolo que tiene el propósito de orientar la transversalización de la perspectiva de género en el diagnóstico, en el contexto de actividades prácticas formativas de la carrera de psicología. Se trató de un estudio de tipo cualitativo con enfoque de corte aplicado que implicó un procedimiento de diseño de este instrumento a partir de la sistematización de documentos curriculares y entrevistas a estudiantes y docentes; así como su posterior examinación por medio de juicios de expertos y retroalimentaciones de estudiantes usuarios. Se encontró que el protocolo apoya al estudiante a identificar los sesgos que pueden tener sus propios prejuicios de género sobre el trabajo diagnóstico. Se concluye que este material contribuye al desarrollo de la competencia de diagnóstico en psicología y que puede ser un recurso valioso para el logro del perfil de egreso de los/as futuros/as profesionales.

\section{Validation protocol to mainstream gender perspective in psychology degree diagnostic practices}

\begin{abstract}
The present study describes the process of design and validation of a protocol aimed to guide gendermainstreaming diagnosis within the context of practical training activities in psychology degrees. This is a qualitative study with an applied cutting-approach that involves a construction-based systematization process of curriculum documentation and professor and student interviews. This is validated by experts' assessments and student-user feedback. The results show that the protocol developed here facilities student identification and awareness about their own biases and gender prejudices in psychological diagnoses. It is concluded that the protocol developed here improves the diagnostic competence of psychology students, improving their professional career profile.
\end{abstract}




\section{INTRODUCCIÓN}

En Chile, a partir del año 2018, el activismo político estudiantil y las inéditas manifestaciones feministas evidenciaron la urgencia que tienen las universidades de hacerse cargo de las brechas entre mujeres y hombres, la violencia de género y el acoso sexual. Con esta intención, las instituciones de educación superior inician la construcción de una agenda de género que recoge las recomendaciones de organismos internacionales como la Organización de las Naciones Unidas para la Educación, la Ciencia y la Cultura (UNESCO), la Organización para la Cooperación y el Desarrollo Económico (OECD) y el Banco Mundial, de tal manera de generar cambios que impliquen, entre otros, la identificación de prejuicios y las modificaciones curriculares necesarias para introducir la perspectiva de género en el análisis para todas las disciplinas (Morley, 2013). La perspectiva de género, que emerge en el marco de la lucha feminista a mediados del siglo $\mathrm{XX}$, se ha considerado como una forma teórica de mirar y de pensar los procesos, las necesidades y las demandas sociales de modo que los análisis permitan entender las desigualdades entre mujeres y hombres. Dicha perspectiva representa el uso de una categoría, el género, como estrategia para observar no solamente las barreras o inequidades que dificultan la igualdad de oportunidades entre los sexos, sino también los mecanismos a través de los cuales las interacciones entre las personas están atravesadas por profundas relaciones de poder enraizadas en estereotipos culturales que estructuran la socialización de género (Chowdhury, 2009).

Ahora bien, ante los intentos de acortar la distancia entre el ámbito de los principios teóricos orientadores y el ámbito de las prácticas sociales concretas, se ha producido un importante debate sobre qué tan transformadora o correctiva es la traducción de las concepciones de relaciones de género justas en una práctica política (Davids et al., 2013). Esta dimensión del problema es crítica para las instituciones de educación superior, donde las acciones de cambio pasan por formas efectivas de institucionalización de la perspectiva de género a través del establecimiento de procedimientos que constituyan comportamientos formalmente reconocidos (Minto y Mergaert, 2018). Uno de estos procedimientos incluye el currículo, y la forma en que los agentes educativos y los tomadores de decisiones inciden desde la perspectiva de género tanto en el currículum explícito como en el denominado "currículum oculto", el cual, por lo general, se encuentra generizado (Ordorika, 2015).

El manuscrito que aquí se presenta se inserta en esta línea de acción universitaria, asumida por la carrera de Psicología de la Universidad Católica de Temuco (UCT), por medio de describir el proceso de diseño y validación de un protocolo que tiene el propósito de orientar la transversalización de la perspectiva de género (TPG) en el proceso diagnóstico, mediante una serie de preguntas e indicaciones, que permitan al estudiante en práctica, emitir una directriz de abordaje pertinente a la dimensión de género del problema que motivó la evaluación. Se trata de un recurso de aprendizaje cuya ventaja es la toma de conciencia por parte del estudiante sobre su concepción del género y las posibilidades que tiene para analizar cómo esta construcción social puede influir en su actuar profesional; a la vez, para los programas de estudios de esta disciplina a nivel pregrado, constituye un material cuyo uso aporta a los perfiles de egreso, específicamente, a la competencia de diagnóstico en Psicología.

A partir de la década de 1990, luego de varias décadas de discusión en distintos espacios sociales y políticos, la comunidad internacional le da mayor preponderancia a la TPG, aprobada como una de las 12 áreas de preocupación de las Naciones Unidas para que sea implementada por sus estados miembros (Kwesiga y Ssendiwala, 2006). Considerando que estas propuestas se inscriben en una agenda por una mayor democratización de las sociedades (Lyle-Gonga, 2013), en las universidades la TPG se torna una opción política para actuar conforme a los principios que orientan el desarrollo democrático (Larrondo y Rivero, 2017).

En las últimas décadas, la idea de TPG ha sido objeto de un profuso debate conceptual entre la teorización académica, el activismo político y su aplicación institucional. Ahora bien, más allá de que pueda ser comprendido en una vertiente liberal como acciones afirmativas, igualdad de trato, igualdad de participación o reformas de gobierno, o en su vertiente radical como crítica cultural profunda, la propuesta del Consejo Económico y Social de las Naciones Unidas ha definido la TPG como el "proceso de evaluación de las consecuencias para las mujeres y los hombres de cualquier actividad planificada, inclusive las leyes, políticas o programas, en todos los sectores y a todos los niveles.

Es una estrategia destinada a hacer que las preocupaciones y experiencias de las mujeres, así como de los hombres, sean un elemento integrante de la elaboración, la aplicación, la supervisión y la evaluación de las políticas y los programas en todas las esferas políticas, económicas y sociales, a fin de que las mujeres y los hombres se beneficien por igual y se impida que se perpetúe la desigualdad" (NU, 1999). Siguiendo a García (2009), la TPG representa una estrategia clave para el cumplimiento de los acuerdos del año 1995 contenidos en la Declaración y la Plataforma de Beijing, que a poco más de dos décadas sigue representando uno de los principales instrumentos de presión del movimiento de mujeres para con las instituciones por medio de las siguientes cuatro definiciones básicas que buscan avanzar en igualdad: género, roles de género, condición y posición social (ver Tabla 1). 


\section{OTROS ANTECEDENTES}

El presente proyecto representa un avance en el ámbito universitario porque se propone la generación de recursos de aprendizaje, en este caso, un protocolo que tribute directamente a la TPG en el currículum (Ordorika, 2015), en específico, en la elaboración de diagnósticos por estudiantes en práctica de la carrera de Psicología de la UCT. La relevancia de relacionar la TPG con la práctica diagnóstica se debe, por un lado, a que esta representa una competencia primordial para el desempeño profesional; $y$, por otro, a que hay un creciente consenso de que el género es un factor asociado a la salud en general y a la salud mental en particular (Montero et al., 2004). Es más, el género es considerado en sí mismo un factor que, en el marco de los determinantes sociales de la salud, puede contribuir a generar o agravar inequidades sanitarias. En efecto, el género es tan relevante para explicar el estado de bienestar de un sujeto que hay consenso en que este explica, al menos en parte, la prevalencia diferencial de diversas enfermedades entre hombres y mujeres; por ejemplo, depresión y ansiedad con más prevalencia entre estas últimas, mientras los trastornos por abuso de sustancias psicotrópicas en los primeros (Mamani et al., 2020), siendo además un factor explicativo de fenómenos como la violencia y la dependencia emocional en pareja (Ramiro-Sánchez et al., 2018a; 2018b).

Tabla 1. Definiciones básicas para la Transversalización de la Perspectiva de Género, según García (2009).

\begin{tabular}{|l|l|}
\hline Concepto & Definición \\
\hline $\begin{array}{l}\text { Sexo- } \\
\text { género }\end{array}$ & $\begin{array}{l}\text { Forma en que cada sociedad determina las funciones, actitudes, valores y relaciones que conciernen a los } \\
\text { hombres y a las mujeres. Mientras el sexo hace referencia a los aspectos biológicos que se derivan de las } \\
\text { diferencias sexuales (macho-hembra), el género hace referencia a una construcción cultural (masculino- } \\
\text { femenino). }\end{array}$ \\
\hline $\begin{array}{l}\text { Roles de } \\
\text { género }\end{array}$ & $\begin{array}{l}\text { Conjunto de tareas y funciones que se asignan a mujeres y hombres en una sociedad dada y en un momento } \\
\text { histórico concreto. Estos roles son aprendidos, se estructuran socialmente y están influidos por factores como la } \\
\text { clase social, la edad, la etnia, etc. Con base en el sexo, se sigue relacionando a las mujeres con el trabajo } \\
\text { reproductivo, y a los hombres con el productivo, lo que también se llamada división sexual del trabajo. }\end{array}$ \\
\hline $\begin{array}{l}\text { Condición } \\
\text { social }\end{array}$ & $\begin{array}{l}\text { Condiciones concretas de vida de mujeres y hombres (por ejemplo, acceso a servicios sanitarios). Las } \\
\text { condiciones se expresan en necesidades de uno u otro sexo. }\end{array}$ \\
\hline $\begin{array}{l}\text { Posición } \\
\text { social }\end{array}$ & $\begin{array}{l}\text { Posición o desigual ubicación social, económica, política y cultural de las mujeres con respecto a los hombres } \\
\text { en un momento histórico determinado. Se manifiesta en los intereses de las mujeres por cambiar su situación } \\
\text { relativa en la sociedad }\end{array}$ \\
\hline
\end{tabular}

El diagnóstico en Psicología requiere de una TPG para responder a su propósito de discernir los fenómenos del bienestar en el contexto amplio de los determinantes sociales de la salud. Como concepto, el diagnóstico tiene larga data en la historia. Del antiguo griego diagignōskein, conjuga el sufijo "dia", que significa "a través de", y "gignōskein" (gnosis), entendido como "conocimiento", y se puede entender de manera literal tanto como "a través del conocimiento" o "discernimiento". En el uso moderno se comprendió bajo la idea de discriminación científica y, por lo común, como el reconocimiento que se hace de una enfermedad a partir de sus síntomas. A principios del siglo $\mathrm{XX}$, el uso psiquiátrico de la noción común de diagnóstico fue problematizada, o al menos comprendida como un proceso, especialmente desde el psicoanálisis (Bonnin, 2014; 2017). Actualmente, el diagnóstico en Psicología es concebido como un proceso que involucra múltiples metodologías e instancias de interacción con la entidad social (individuos, escuelas, procesos organizacionales, comunidades) a ser diagnosticada (Bonnin, 2013; Woolgar y Scott, 2014). Y si bien, en las carreras de Psicología existe una amplia variedad de contextos de práctica profesional (clínicos, educativos, organizacionales y social-comunitarios) y múltiples ámbitos temáticos de trabajo, es posible, de modo general, comprender el proceso diagnóstico como un procedimiento que cumple una sucesión programada de etapas.

Con base en la revisión de Capurro y Rada (2007) y Blanco et al. (2010) se puede observar que son cuatro las fases de un diagnóstico: primero, una fase preliminar donde se establece el tipo de relación con el objeto diagnóstico y el encuadre entre la demanda de la institución y la del beneficiario/paciente. Una segunda fase donde se produce la primera interacción con el sujeto diagnóstico, y cuyo propósito es recolectar los primeros antecedentes e información secundaria pertinente. En la tercera fase se desarrolla la recolección de nueva información, según la hipótesis inicial, y se analiza la nueva información (por ejemplo, por medio de tests). Por último, en la cuarta fase, se genera la emisión de un diagnóstico y la elección de intervención o plan de acción/estrategia de solución. En la Tabla 2, se presentan los detalles de las etapas del proceso diagnóstico en las Ciencias Sociales y de la Salud.

El protocolo cuyo proceso de diseño y validación se detalla se basa en estos antecedentes para avanzar en la dirección de la TPG en el diagnóstico en el contexto de prácticas de la carrera de Psicología. Dicho de otro modo, la intención es generar un instrumento para el/la estudiante que le guíe en su aprendizaje mediante una serie de preguntas e indicaciones de tal manera que sea capaz de construir una directriz de abordaje pertinente en términos de género al problema que motivó la evaluación diagnóstica en el contexto de las actividades formativas del pregrado. En el apartado siguiente se presenta la metodología del estudio y, posteriormente, los resultados. 
Tabla 2. Etapas del proceso diagnóstico.

\begin{tabular}{|l|l|l|l|}
\hline Fases & $\begin{array}{l}\text { Salud clínica pública (Cuba) (Blanco, } \\
\text { et al., 2010) }\end{array}$ & $\begin{array}{l}\text { Salud clínica (Capurro y Rada, } \\
\text { 2007) }\end{array}$ & Proceso diagnóstico \\
\hline I & --- & ---- & $\begin{array}{l}\text { Etapa preliminar. Establecimiento } \\
\text { del tipo de relación con el objeto de } \\
\text { estudio/diagnóstico }\end{array}$ \\
\hline II & $\begin{array}{l}\text { Revisión de antecedentes y } \\
\text { documentos previos. } \\
\text { Argumentación de las hipótesis. } \\
\text { Comparación de hipótesis entre sí } \\
\text { (diagnóstico diferencial). }\end{array}$ & $\begin{array}{l}\text { Observación preliminar. Primer } \\
\text { momento en la generación de } \\
\text { hipótesis diagnósticas. }\end{array}$ & $\begin{array}{l}\text { Primera interacción con el objeto de } \\
\text { estudio/diagnóstico. Información } \\
\text { secundaria/antecedentes }\end{array}$ \\
\hline III & $\begin{array}{l}\text { Selección de nuevos estudios a } \\
\text { indicación e interpretación de los } \\
\text { resultados. }\end{array}$ & $\begin{array}{l}\text { Test: nueva información (síntomas, } \\
\text { signos o exámenes adicionales). } \\
\text { Refinamiento de las hipótesis } \\
\text { diagnósticas. }\end{array}$ & $\begin{array}{l}\text { Recolección/test, nueva información } \\
\text { según hipótesis pretest. Análisis de } \\
\text { nueva información recolectada. }\end{array}$ \\
\hline IV & $\begin{array}{l}\text { Comprobación de la hipótesis } \\
\text { diagnóstica. }\end{array}$ & $\begin{array}{l}\text { Verificación del diagnóstico para } \\
\text { iniciar tratamiento. }\end{array}$ & $\begin{array}{l}\text { Comprobación, verificación } \\
\text { diagnóstica. Elección de } \\
\text { tratamiento, plan de } \\
\text { acción/estrategia de solución. }\end{array}$ \\
\hline
\end{tabular}

\section{MÉTODO}

Con la finalidad de diseñar y validar el protocolo para la TPG en el proceso diagnóstico en el contexto de prácticas formativas de estudiantes de pregrado de la carrera de Psicología de la UCT, se recurrió a una metodología de tipo cualitativo con un enfoque de corte aplicado, es decir, que busca resolver o abordar problemas prácticos. La intención fue lograr el diseño y validación de un instrumento tipo protocolo, el cual, según Moher et al. (2015), es un elemento central que permite reducir drásticamente la arbitrariedad en la toma de decisiones y prever problemas potenciales, en este caso, en el ámbito de la evaluación realizada por el/la estudiante en práctica a un/a usuario/a (persona, grupo, organización o comunidad). Este proceso implicó desarrollar un procedimiento secuencial detallado que facilita realizar un diagnóstico que cumpla con el principio de identificar la situación particular de mujeres y hombres considerando sus diferentes realidades y necesidades.

El procedimiento inició con una sistematización que buscó organizar los resultados de experiencias y/o instrumentos similares (Acosta, 2004), en este caso, sobre la TPG en documentos o registros de prácticas en la misma carrera, tales como perfil de egreso, programas de los cursos de niveles intermedios o profesionales, criterios de evaluación de aprendizaje y reglamentos. La información se trianguló con datos recabados por medio de una entrevista grupal, semi-estructurada, dirigida a 8 estudiantes de Psicología, quienes ya habían cursado sus prácticas iniciales y/o profesionales, y a quienes se les consultó sobre la pertinencia y recursos de aprendizaje disponibles a lo largo de su itinerario formativo para incluir la categoría de género como herramienta analítica para el ejercicio profesional. También se generaron datos a partir de la aplicación de un cuestionario con preguntas abiertas dirigido a 4 docentes que habían impartido clases en cursos de prácticas profesionales, quienes respondieron si habían usado la categoría de género en el trabajo con sus estudiantes. A partir de estos resultados se definieron los elementos y criterios centrales que debían estar presentes en el instrumento y se procedió a su diseño.

Posteriormente, se utilizó la técnica del juicio de expertos, la cual permite obtener de una serie de personas su juicio u opinión con respecto a un tema en particular (Galicia et al., 2017). Para ello se seleccionaron tres expertas/os (dos mujeres y un hombre) cuyo perfil se definió como profesional del área de las Ciencias Sociales (psicólogo/a o trabajador/a social), con al menos el grado de magíster y una experiencia o trayectoria profesional o de investigación con perspectiva de género demostrada. El juicio de las y los expertos se realizó mediante un instrumento de validación del contenido que, a partir de una pauta, solicitó que por cada fase del diagnóstico identificada en el protocolo se señalara si se cumplían los siguientes criterios: claridad en la redacción, lenguaje adecuado al nivel del estudiante, pertinencia y cantidad de preguntas; y, sugerencias u observaciones generales.

Finalmente se solicitó a tres estudiantes en práctica del pregrado de Psicología que participaran en la evaluación del protocolo; estos estudiantes fueron dos mujeres y un hombre, de cuarto año, voluntarios, que habían recién concluido sus prácticas intermedias en las que habían realizado diagnósticos con pacientes simulados. En primer lugar, se les explicaron los objetivos del proyecto y se acordó el procedimiento de trabajo entregando el protocolo para una primera lectura. En segundo lugar, se les solicitó en una reunión grupal repetir el procedimiento diagnóstico que ya habían finalizado, pero incorporando estos lineamientos, 
señalándoles que esto no modificaría la calificación que habían obtenido, ni los resultados del diagnóstico; con estas decisiones se evitó que el/la estudiante se viera envuelto en un conflicto de interés que pudiera perjudicar su proceso de aprendizaje o el proceso de validación del instrumento. Posterior a la aplicación del protocolo, los/as estudiantes entregaron sus apreciaciones por escrito por medio de una pauta de validación de contenido, donde reportaron, primero, con respecto a las orientaciones para cada fase del diagnóstico, su claridad en la redacción, lenguaje adecuado, pertinencia temática y de cantidad de indicaciones y, sugerencias u observaciones generales; $y$, segundo, los aprendizajes sobre el procedimiento diagnóstico que habían logrado a partir de considerar la TPG. Como último momento, se realizó una reunión más con los/as estudiantes para aclarar las últimas dudas del equipo investigador. Con ambas validaciones, la de jueces expertos y estudiantado, se ajustó el instrumento y se realizó el diseño final del protocolo. En el apartado siguiente se presenta este, así como una síntesis de los resultados.

\section{RESULTADOS Y DISCUSIÓN}

Con respecto a la dimensión de género como herramienta de estudio en los procesos de prácticas intermedias o profesionales, a través de la sistematización se encontró que la TPG en el currículum explícito se apoya en el principio transversal de las carreras de pregrado de la UCT, según el cual cada estudiante debe aprender desde el marco de referencia de la inclusión, el que otorga énfasis al respeto y la valoración por la diversidad humana y cultural (Herrera-Seda et al., 2016). No obstante, en los documentos base del itinerario, tales como perfil de egreso, programas de estudio y criterios de evaluación se observó que no se declara la dimensión de género como un elemento explícito a estudiar dentro de esta variabilidad de los grupos sociales. Esta apreciación fue corroborada por los/as estudiantes, quienes en su entrevista declararon que la categoría de género no es un elemento que se considere en su formación formal, siendo incluido en algunas materias según la sensibilidad del profesor. Así mismo, los/as docentes expusieron la invisibilización de la TPG en las acciones educativas de los/as futuros psicólogos/as y, en específico, en los procesos de diagnóstico e intervención, tanto a nivel individual como grupal.

Con base en esta información, el equipo de investigación tomó dos decisiones: i. operacionalizar el proceso diagnóstico por medio de las cuatro fases identificadas a partir de la revisión bibliográfica realizada previamente (ver Tabla 2), las que se convirtieron en el eje estructural para la TPG por el/la estudiante; y, ii. operacionalizar la TPG por medio de los siguientes cuatro conceptos o dimensiones: sexo-género, roles de género, condición y posición social (García, 2009, ver Tabla 1). Dichos conceptos permiten al estudiante un acercamiento inicial al uso del género como categoría analítica, permitiendo que este/esta los comprenda a partir de antecedentes teóricos provenientes del campo de la Psicología, tales como rol, condición y posición social, a los que ahora sumaría la distinción entre sexo-género, que es la base de entrada para la transversalización (García, 2009). Se generó entonces un instrumento donde a través de preguntas e indicaciones para cada fase del proceso diagnóstico se invita al estudiante a reflexionar desde una lógica de género, basada en los cuatro conceptos mencionados. El instrumento diseñado se organizó en una matriz para su mejor operatividad, dando espacios para que cada estudiante reportara la aplicación o no de los criterios recomendados, así como las observaciones, dudas o comentarios que pudieran surgir.

Concluido el anterior momento, el protocolo pasó a revisión por jueces expertos. Estos recomendaron enfatizar en el instrumento la orientación al estudiante para que visualice el impacto del género en las historias de vida de los sujetos individuales y, en el comportamiento grupal, cuando se trata de sujetos colectivos (grupos o comunidades). Se observó por los jueces la relevancia de que el/la estudiante reflexionara sobre las implicancias que tiene la sujeción a los estereotipos de género. Lo anterior, ya que la relación entre el género y las historias de vida de mujeres y hombres supone un proceso de autodefinición e individualización que, para el caso de las primeras a diferencia de los segundos, se construye como un "ser para otros", es decir, vinculado al cuidado de la familia y los hijos/as (Mayobre, 2007), con las consecuencias correspondientes con relación a la división sexual del trabajo y la posición jerárquica entre los géneros. Estos elementos se transversalizaron en el protocolo, el cual insta a observar, por ejemplo, las consecuencias de la asignación social de tareas para ambos sexos sobre la situación que motivó el diagnóstico.

Complementariamente, los jueces remarcaron la necesidad de inducir la reflexión del/la estudiante sobre las implicancias para las personas en función de no adecuarse a las normas o comportamientos sociales adecuados a su sexo, por ejemplo, a las expectativas sociales derivadas de la maternidad, con las consecuentes contradicciones entre el rol asignado y el rol desempeñado. Se asumieron estas observaciones, haciendo énfasis en las historias de vida del sujeto, de tal manera de evidenciar las implicaciones del género en las trayectorias vitales 0 , tratándose de grupos o comunidades, en las condiciones sistemáticas de las mujeres y los hombres que los conforman (por ejemplo, se agregaron indicaciones que alientan a explorar las implicaciones de las transgresiones del rol de género). Una síntesis general de las observaciones y decisiones tomadas a partir de la validación por jueces expertos se presenta en la Tabla 3. 
Tabla 3. Síntesis de resultados de la validación por jueces expertos.

\begin{tabular}{|l|l|l|}
\hline $\begin{array}{l}\text { Valoraciones } \\
\text { positivas }\end{array}$ & Recomendaciones & Decisiones tomadas \\
\hline $\begin{array}{l}\text { Acuerdo general } \\
\text { sobre la pertinencia } \\
\text { y la adecuación en } \\
\text { la cantidad de } \\
\text { preguntas. }\end{array}$ & $\begin{array}{l}\text { Enfatizar la perspectiva de género en las } \\
\text { historias de vida de los sujetos individuales y en } \\
\text { las relaciones a nivel de sistema, cuando se } \\
\text { trata de grupos y comunidades. }\end{array}$ & $\begin{array}{l}\text { Se alude al momento actual, pero también a la } \\
\text { historia anterior del sujeto, sobre todo en la fase II } \\
\text { del proceso diagnóstico. }\end{array}$ \\
\cline { 2 - 3 } & $\begin{array}{l}\text { Incluir elementos que ayuden a reflexionar } \\
\text { sobre las implicaciones que tiene para las } \\
\text { personas no responder a los roles de género } \\
\text { socialmente hegemónicos. }\end{array}$ & $\begin{array}{l}\text { Se insta a reflexionar sobre las implicaciones del } \\
\text { sistema sexo-género en el problema que motivó el } \\
\text { diagnóstico. Se hace referencia explícita a las } \\
\text { transgresiones de los roles de género en la fase I } \\
\text { del proceso diagnóstico. }\end{array}$ \\
\cline { 2 - 3 } & $\begin{array}{l}\text { Clarificar conceptos teóricos, de tal manera que } \\
\text { el/la estudiante pueda guiarse en la aplicación } \\
\text { del protocolo por definiciones básicas. }\end{array}$ & $\begin{array}{l}\text { Se agregó en el protocolo la columna de dimensión, } \\
\text { de tal manera que el/la estudiante supiera a qué } \\
\text { elemento teórico refería cada pregunta e indicación. }\end{array}$ \\
\cline { 2 - 3 } & $\begin{array}{l}\text { Mejorar la redacción de ciertas preguntas para } \\
\text { adecuarlas al lenguaje a un estudiante en } \\
\text { formación. }\end{array}$ & Se aceptan las recomendaciones. \\
\hline
\end{tabular}

Sobre los resultados de la validación por estudiantes, se destaca la valoración positiva que realizaron respecto al instrumento al referir que les sensibilizó en relación con las implicaciones del género sobre los problemas y situaciones que enfrentan los/as usuarios/as. Que el estudiantado participante haya reconocido este aspecto resulta de relevancia ya que la literatura es coincidente sobre que, en Chile y en general en los países occidentales, siguen operando patrones culturales entre los jóvenes que reproducen estereotipos y representaciones tradicionales de género que perpetúan la discriminación y la inequidad entre los sexos (Cubillas et al. 2016; García y Castro 2017; Maes et al. 2019). Dicho en otras palabras, el/la estudiante requiere de un apoyo en su formación para que sea capaz de identificar los sesgos que podrían tener sus propias orientaciones y prejuicios de género sobre el trabajo diagnóstico.

Otro elemento mencionado por los/as estudiantes en su retroalimentación al instrumento tuvo relación con la necesidad de reflexionar sobre la relevancia de usar instrumentos que sean sensibles al género; en este sentido, fueron capaces de reconocer que, a pesar del avance teórico y metodológico del concepto de género en el ámbito de la salud, que ha derivado en nuevas prácticas, manuales, guías, protocolos, etc., la relación entre género y evaluación continua siendo limitada, en cuanto no ha sido aún suficientemente estudiada y estructurada (Gambara, 2012). Por eso fue importante incluir una pregunta e indicación en la última fase del diagnóstico donde el/la estudiante reconoce las limitaciones que tuvo este proceso, si las hubo, por motivos de la carencia en la disciplina de la Psicología de instrumentos de evaluación que cumplan con los criterios para la TPG. De esta manera, se alienta al/la estudiante a hacerse cargo de las limitaciones tanto de su formación como del campo disciplinar en el cual se está formando. El fin último de esto es alentarle a que, llegado el momento, genere las condiciones para que se continúe la atención del sujeto con base en una ampliación de la comprensión de su problema en términos de género. Una síntesis de los resultados de esta etapa del procedimiento se muestra en la Tabla 4.

Tabla 4. Síntesis de resultados de la validación por el estudiantado.

\begin{tabular}{|l|l|l|}
\hline Validaciones positivas & Recomendaciones & Decisiones tomadas \\
\hline $\begin{array}{l}\text { Acuerdo general sobre la } \\
\text { pertinencia y la adecuación en } \\
\text { la cantidad de preguntas. }\end{array}$ & $\begin{array}{l}\text { Incorporar preguntas e indicaciones que } \\
\text { orienten al estudiante para aprender a } \\
\text { distinguir casos en los que el sistema sexo- } \\
\text { género es primordial en la definición del } \\
\text { problema que motivó el diagnóstico. }\end{array}$ & $\begin{array}{l}\text { Se incorpora una pregunta e indicación } \\
\text { como último punto de la fase I que orienta la } \\
\text { decisión de continuar o no con el protocolo } \\
\text { de acuerdo con su relevancia para la } \\
\text { comprensión del sujeto. }\end{array}$ \\
\hline $\begin{array}{l}\text { Desarrollo de sensibilización } \\
\text { sobre las implicaciones que el } \\
\text { sistema sexo-género tiene para } \\
\text { el bienestar de los/as } \\
\text { usuarios/as. }\end{array}$ & $\begin{array}{l}\text { Incorporar indicaciones frente a la emisión } \\
\text { de un diagnóstico o elección de intervención } \\
\text { limitado por elementos teóricos o } \\
\text { instrumentos metodológicos no sensibles a a } \\
\text { la perspectiva de género. }\end{array}$ & $\begin{array}{l}\text { Se incorpora una pregunta e indicación } \\
\text { como último punto de la fase IV que orienta } \\
\text { la decisión de declarar las limitaciones del } \\
\text { proceso y, en su caso, recomendaciones } \\
\text { para un proceso posterior. }\end{array}$ \\
\hline $\begin{array}{l}\text { Desarrollo de elementos } \\
\text { teóricos y metodológicos que } \\
\text { permiten un abordaje del ser } \\
\text { humano en su complejidad } \\
\text { biopsicosocial. }\end{array}$ & $\begin{array}{l}\text { Incorporar el protocolo en la formación del } \\
\text { pregrado ya que esta, a su juicio, no } \\
\text { proporciona al estudiante instancias } \\
\text { formativas en temas de género ni en } \\
\text { procedimientos para la incorporación de la } \\
\text { perspectiva en la actuación profesional. }\end{array}$ & $\begin{array}{l}\text { Dar continuidad al proceso, presentando el } \\
\text { material generado ante los/as docentes guía } \\
\text { de prácticas para su retroalimentación. } \\
\text { Publicar el material de tal manera de que } \\
\text { sea un material complementario para la } \\
\text { aplicación del protocolo. }\end{array}$ \\
\hline
\end{tabular}


El protocolo en su versión final se muestra en la Tabla 5. Como instrumento diseñado y validado permite al estudiante sensibilizarse sobre las limitaciones que en términos de prejuicios de género puede mostrar, muy posiblemente por la etapa de desarrollo que atraviesa (Cubillas et al. 2016; García y Castro 2017). El protocolo tributa a pensar el desarrollo de apoyos para el aprendizaje que consideren a cada estudiante de modo integral, es decir, no solo en su rol frente al docente, sino también como persona perteneciente a un rango etario y una condición genérica. En este escenario, como recurso de aprendizaje responde a los lineamientos de diversos organismos internacionales para la identificación de prejuicios y el desarrollo de las modificaciones curriculares que sean necesarias para introducir el género como categoría de análisis en la disciplina y la formación universitaria a nivel de currículum del pregrado de Psicología (Morley, 2013; Buquet, 2011), en este caso, a partir de un material que favorece la metacognición estudiantil.

La universidad como institución de formación en estudios superiores representa un espacio idóneo para la implementación y desarrollo de la TPG, lo cual requiere llevar a cabo una serie de transformaciones estructurales, siendo el currículum de cada carrera una pieza clave en dicho proceso. El presente trabajo representa una contribución en este sentido, pensando desde las necesidades del pregrado de Psicología, generando un material que posibilita intervenir en una etapa crítica de la formación con miras a atenuar el potencial sesgo y estereotipos de género que, debido a su propia socialización de género, el/la estudiante en práctica pudiera adoptar en el ejercicio diagnóstico. Así, el protocolo que se presenta constituye una herramienta para integrar la perspectiva de género como punto de referencia evaluativo de los roles, biografías y situaciones sociales.

Este primer paso obliga a pensar en el desafío mayor que significa la integración curricular transversal de la perspectiva de género en la disciplina. Se trata de una tarea con grandes pendientes aún para la Psicología, sobre todo porque implica un punto de intersección disciplinar, en este caso, con los Estudios de Género. A la vez, la amplia heterogeneidad de paradigmas y marcos epistémicos psicológicos representan una oportunidad para que la perspectiva de género se integre transversalmente al currículo.

Tabla 5. Protocolo para transversalizar la perspectiva de género en el diagnóstico en contexto de prácticas de pregrado de la carrera de Psicología.

\begin{tabular}{|c|c|c|c|c|}
\hline $\begin{array}{l}\text { Fases del proceso } \\
\text { diagnóstico }\end{array}$ & Preguntas e indicaciones & Dimensión & $\begin{array}{l}\text { Marque una } X \text { si } \\
\text { consideró estos } \\
\text { elementos en el } \\
\text { proceso diagnóstico }\end{array}$ & $\begin{array}{l}\text { Observaciones o } \\
\text { comentarios en } \\
\text { relación con el } \\
\text { proceso diagnóstico }\end{array}$ \\
\hline \multirow{7}{*}{$\begin{array}{l}\text { I. Etapa preliminar. } \\
\text { Propósito: Establecer } \\
\text { el tipo de relación con } \\
\text { la persona, grupo o } \\
\text { comunidad que es el } \\
\text { foco del proceso } \\
\text { diagnóstico } \\
\text { (usuario/a). }\end{array}$} & $\begin{array}{l}\text { ¿Puedo distinguir la forma en la que el/la usuario/a } \\
\text { expresa su sexo y género? }\end{array}$ & \multirow[t]{2}{*}{$\begin{array}{l}\text { Sexo- } \\
\text { Género }\end{array}$} & & \\
\hline & $\begin{array}{l}\text { Indicaciones: Si es un/a usuario/a individual: identifico } \\
\text { su expresión en términos de sexo y su género. Si es } \\
\text { un grupo o comunidad: identifico su composición en } \\
\text { términos de mujeres y hombres. }\end{array}$ & & & \\
\hline & $\begin{array}{l}\text { ¿En qué medida el/la usuario/a, en razón de la lógica } \\
\text { sexo-género, define el contrato de trabajo? }\end{array}$ & \multirow[t]{2}{*}{$\begin{array}{l}\text { Roles } \\
\text { Condición }\end{array}$} & & \\
\hline & $\begin{array}{l}\text { Indicaciones: Si es un usuario/a individual: identifico la } \\
\text { posición social que ocupa como mujer u hombre, y } \\
\text { doy cuenta de cómo esta posición tiene implicancias } \\
\text { sobre los propósitos y los medios del proceso } \\
\text { diagnóstico. Si es un grupo o comunidad: Identifico } \\
\text { las brechas de género presentes en el grupo o } \\
\text { comunidad y doy cuenta de las implicancias de ello } \\
\text { sobre la definición de los propósitos y los medios del } \\
\text { proceso diagnóstico. }\end{array}$ & & & \\
\hline & $\begin{array}{l}\text { ¿De qué manera mi condición de sexo-género tiene } \\
\text { implicancias sobre el usuario/a y, en general, sobre la } \\
\text { situación diagnóstica? }\end{array}$ & \multirow[t]{2}{*}{ Condición } & & \\
\hline & $\begin{array}{l}\text { Indicaciones: Identifico de qué manera mi sexo- } \\
\text { género facilita, obstaculiza o limita el proceso } \\
\text { diagnóstico. }\end{array}$ & & & \\
\hline & $\begin{array}{l}\text { De acuerdo con mis apreciaciones preliminares, ¿es } \\
\text { relevante para la comprensión del sujeto incorporar la } \\
\text { perspectiva de género en el proceso diagnóstico? Sí o } \\
\text { no. }\end{array}$ & $\begin{array}{l}\text { Toma de } \\
\text { decisión: } \\
\text { Activación } \\
\text { formal del } \\
\text { protocolo. }\end{array}$ & & \\
\hline
\end{tabular}


Tabla 5: continuación.

\begin{tabular}{|c|c|c|c|c|}
\hline $\begin{array}{l}\text { Fases del proceso } \\
\text { diagnóstico }\end{array}$ & Preguntas e indicaciones & Dimensión & $\begin{array}{l}\text { Marque una } X \text { si } \\
\text { consideró estos } \\
\text { elementos en el } \\
\text { proceso } \\
\text { diagnóstico }\end{array}$ & $\begin{array}{l}\text { Observaciones o } \\
\text { comentarios en } \\
\text { relación con el } \\
\text { proceso diagnóstico }\end{array}$ \\
\hline \multirow{4}{*}{$\begin{array}{l}\text { II. Primera interacción } \\
\text { con el sujeto } \\
\text { diagnóstico. } \\
\text { Propósito: Recolectar } \\
\text { primeros antecedentes } \\
\text { e información } \\
\text { secundaria pertinente. }\end{array}$} & $\begin{array}{l}\text { ¿Puedo diferenciar, con base en una lógica de } \\
\text { género, las necesidades y las demandas del } \\
\text { usuario/a y su relación con las demandas que } \\
\text { plantea frente al proceso diagnóstico? }\end{array}$ & \multirow[t]{2}{*}{$\begin{array}{l}\text { Roles } \\
\text { Condición }\end{array}$} & & \\
\hline & $\begin{array}{l}\text { Indicaciones: Si es un usuario/a individual: Identifico } \\
\text { sus necesidades y demandas y las interpreto en } \\
\text { función del rol de género que ocupa o de las } \\
\text { transgresiones a este rol que le ha sido asignado } \\
\text { socialmente. Si es un grupo y/o comunidad: Identifico } \\
\text { necesidades y demandas diferenciadas entre } \\
\text { mujeres y hombres y su relación con las brechas de } \\
\text { género detectadas en el grupo o comunidad. }\end{array}$ & & & \\
\hline & $\begin{array}{l}\text { ¿Qué papeles están asignados al usuario/a en } \\
\text { términos de género y cómo esta situación se } \\
\text { relaciona con el problema que motivó el diagnóstico? }\end{array}$ & \multirow[t]{2}{*}{ Roles } & & \\
\hline & $\begin{array}{l}\text { Indicaciones: Reflexiono si el problema que origina el } \\
\text { diagnóstico es consecuencia, al menos parcial, de } \\
\text { una división sexual del trabajo que conlleva } \\
\text { inequidades, barreras y obstáculos para uno u otro } \\
\text { sexo en cierto ámbito; o bien, si es consecuencia, al } \\
\text { menos parcial, de las transgresiones del usuario/a } \\
\text { test los roles de género establecidos. }\end{array}$ & & & \\
\hline \multirow{6}{*}{$\begin{array}{l}\text { III. Recolección de } \\
\text { nueva información, } \\
\text { según hipótesis inicial. } \\
\text { Propósito: Recolectar y } \\
\text { analizar nueva } \\
\text { información (por } \\
\text { ejemplo, por medio de } \\
\text { test). }\end{array}$} & $\begin{array}{l}\text { ¿Puedo distinguir de qué manera la lógica de sexo- } \\
\text { género del usuario/a se relaciona con sus } \\
\text { condiciones materiales? }\end{array}$ & \multirow[t]{2}{*}{$\begin{array}{l}\text { Roles } \\
\text { Condición }\end{array}$} & & \\
\hline & $\begin{array}{l}\text { Indicaciones: Identifico la manera en que ser mujer o } \\
\text { ser hombre ha implicado, actualmente o en la historia } \\
\text { anterior, barreras o inequidades materiales. }\end{array}$ & & & \\
\hline & $\begin{array}{l}\text { ¿A qué recursos accede el/la usuario/a y qué } \\
\text { recursos controla en términos de género? }\end{array}$ & \multirow[t]{2}{*}{ Posición } & & \\
\hline & $\begin{array}{l}\text { Indicaciones: Observo si el problema que origina el } \\
\text { diagnóstico es consecuencia, al menos parcial, de } \\
\text { una inequidad en el acceso y/o el control de los } \\
\text { recursos por razones de sexo y/o género. }\end{array}$ & & & \\
\hline & $\begin{array}{l}\text { ¿Qué decisiones toma el/la usuario/a, en qué lugares } \\
\text { y espacios y de qué manera esta dinámica se } \\
\text { relaciona con el problema a abordar? }\end{array}$ & \multirow[t]{2}{*}{$\begin{array}{l}\text { Roles } \\
\text { Condición }\end{array}$} & & \\
\hline & $\begin{array}{l}\text { Indicaciones: Observo si el problema que origina el } \\
\text { diagnóstico se relaciona con obstáculos o } \\
\text { inequidades en la participación del usuario/a en } \\
\text { términos del sexo y/o género. }\end{array}$ & & & \\
\hline \multirow{6}{*}{$\begin{array}{l}\text { IV. Emisión de } \\
\text { diagnóstico y elección } \\
\text { de intervención, plan de } \\
\text { acción/estrategia de } \\
\text { solución. } \\
\text { Propósito: Emitir el } \\
\text { diagnóstico e indicar } \\
\text { una estrategia de } \\
\text { solución. }\end{array}$} & $\begin{array}{l}\text { ¿Soy capaz de emitir un diagnóstico que dé cuenta } \\
\text { de la relación entre el problema de estudio y la } \\
\text { condición de sexo-género del usuario/a? }\end{array}$ & \multirow[t]{2}{*}{$\begin{array}{l}\text { Sexo- } \\
\text { Género }\end{array}$} & & \\
\hline & $\begin{array}{l}\text { Indicaciones: Identifico de qué manera y con qué } \\
\text { relevancia la condición de sexo-género del usuario/a } \\
\text { se relaciona con la problemática de interés en el } \\
\text { proceso diagnóstico. }\end{array}$ & & & \\
\hline & $\begin{array}{l}\text { ¿De qué manera el plan de intervención involucra la } \\
\text { participación del usuario/a según su posición sexo- } \\
\text { género? }\end{array}$ & \multirow[t]{2}{*}{$\begin{array}{l}\text { Roles } \\
\text { Condición } \\
\text { Posición }\end{array}$} & & \\
\hline & $\begin{array}{l}\text { Indicaciones: Considero en el diseño del plan de } \\
\text { intervención instancias de co-construcción de metas } \\
\text { y propósitos con el/la usuario/a afines a su posición } \\
\text { de género. }\end{array}$ & & & \\
\hline & $\begin{array}{l}\text { ¿Cómo impacta el plan de intervención en el papel, } \\
\text { los recursos y las necesidades y demandas del } \\
\text { usuario/a en términos de género? }\end{array}$ & \multirow[t]{2}{*}{$\begin{array}{l}\text { Roles } \\
\text { Condición } \\
\text { Posición }\end{array}$} & & \\
\hline & $\begin{array}{l}\text { Indicaciones: Identifico las implicancias que tiene el } \\
\text { plan de intervención sobre los obstáculos y/o } \\
\text { inequidades de género relacionadas a la situación } \\
\text { diagnosticada. }\end{array}$ & & & \\
\hline
\end{tabular}


Tabla 5: continuación.

\begin{tabular}{|c|c|c|c|c|}
\hline $\begin{array}{c}\text { Fases del proceso } \\
\text { diagnóstico }\end{array}$ & Preguntas e indicaciones & Dimensión & $\begin{array}{l}\text { Marque una } X \text { si } \\
\text { consideró estos } \\
\text { elementos en el } \\
\text { proceso diagnóstico }\end{array}$ & $\begin{array}{l}\text { Observaciones o } \\
\text { comentarios en } \\
\text { relación con el } \\
\text { proceso diagnóstico }\end{array}$ \\
\hline \multirow{2}{*}{$\begin{array}{l}\text { IV. Emisión de } \\
\text { diagnóstico y elección } \\
\text { de intervención, plan de } \\
\text { acción/estrategia de } \\
\text { solución. }\end{array}$} & $\begin{array}{l}\text { ¿En qué sentidos la solución al problema } \\
\text { diagnosticado involucra cambios a favor de la } \\
\text { igualdad de género? De ser así, ¿en qué consisten } \\
\text { estos cambios? }\end{array}$ & \multirow[t]{2}{*}{$\begin{array}{l}\text { Roles } \\
\text { Condición } \\
\text { Posición }\end{array}$} & & \\
\hline & $\begin{array}{l}\text { Indicaciones: Diseño un plan que permite avanzar } \\
\text { en términos de equidad de género como estrategia } \\
\text { para superar el problema que motivó el diagnóstico. }\end{array}$ & & & \\
\hline \multirow{2}{*}{$\begin{array}{l}\text { Propósito: Emitir el } \\
\text { diagnóstico e indicar } \\
\text { una estrategia de } \\
\text { solución. }\end{array}$} & $\begin{array}{l}\text { ¿Cuáles son las limitaciones del proceso } \\
\text { diagnóstico en términos de transversalización de la } \\
\text { perspectiva de género y qué implicaciones tienen } \\
\text { estas limitaciones para el sujeto? }\end{array}$ & \multirow[t]{2}{*}{$\begin{array}{l}\text { Sexo- } \\
\text { Género }\end{array}$} & & \\
\hline & $\begin{array}{l}\text { Indicaciones: Declaro en el informe, si el diagnóstico } \\
\text { tiene limitaciones en su transversalización del } \\
\text { enfoque de género (por ej. uso de pruebas no } \\
\text { estandarizadas para personas con orientación no } \\
\text { heterosexual), sus implicaciones en el juicio y la } \\
\text { elección de tratamiento y, por último, las } \\
\text { recomendaciones que otras acciones profesionales } \\
\text { deben considerar para superarlas. }\end{array}$ & & & \\
\hline
\end{tabular}

Surge para esto la exigencia de llevar a cabo un ordenamiento interdisciplinar sistemático que logre establecer la coherencia analítica entre, por un lado, las Ciencias de la Salud, destacando allí como principal instancia el quehacer clínico; y por otro lado, las Ciencias Sociales y de Género, especialmente, los enfoques postestructuralistas de la identidad y la acción social. Esta tarea de integración curricular permitirá trazar potencialidades y limitaciones de la perspectiva de género para la disciplina, e igualmente en el sentido inverso, reconocer las potencialidades y limitaciones que la disciplina impone a la TPG.

El procedimiento metodológico seguido permite su replicación con propósitos equivalentes, por ejemplo, en la validación de protocolos que permitan al estudiante responder con pertinencia a las demandas y necesidades de otros grupos socioeconómica y geográficamente vulnerables (por ejemplo, disidencias sexuales o grupos indígenas). De esta manera, se pone a disposición de la comunidad científica una metodología para avanzar en la TPG a nivel currículum y, en paralelo, responder a la demanda estudiantil por la igualdad y la equidad entre las diversas identidades que confluyen en el ambiente universitario y las prácticas profesionales de la disciplina.

\section{CONCLUSIONES}

El protocolo de diagnóstico desarrollado para estudiantes en práctica de la carrera de psicología de la UCT, constituye un intento por avanzar en la TPG en el espacio universitario. La construcción de este instrumento se desarrolló para apoyar la formación de los estudiantes de pregrado mediante una herramienta práctica en cuanto facilita, mediante la entrega de directrices en la intervención psicosocial elementos teóricos que permiten reflexionar sobre el impacto diferenciador de la perspectiva de género en los usuarios/as a intervenir. Debido a que tributa a la competencia de diagnóstico en Psicología, el uso de este recurso lo hace también al perfil de egreso de los profesionales de este campo.

\section{REFERENCIAS}

Acosta, L., Guía práctica para la sistematización de proyectos y programas de cooperación técnica. FAO, (2004).

Blanco M, Morales H., y Rodríguez T., Actividad, acciones y operaciones en el proceso diagnóstico, educación médica superior, 24(3), 352-359 (2010).

Bonnin, J., The public, the private and the intimate in doctor-patient communication: admission interviews at an outpatient mental health care service, https://doi.org/10.1177/1461445613492249, Discourse Studies, 15(6), 687-711 (2013).

Bonnin, J., Treating without diagnosis: psychoanalysis in medical settings in Argentina, https://doi.org/10.1558/cam.v11i1.26446, Communication and Medicine, 11(1), 15-26 (2014).

Bonnin, J., Formulations in psychotherapy: admission interviews and the conversational construction of diagnosis, https://doi.org/10.1177/1049732316686333, Qualitative Health Research, 27(11), 1591-1599 (2017).

Buquet, A., Transversalización de la perspectiva de género en la educación superior: problemas conceptuales y prácticos, Perfiles Educativos, 33, 221-225 (2011). 
Capurro D., y Rada G, The diagnostic process, http://dx.doi.org/10.4067/S0034-98872007000400018, Revista Médica de Chile, 135(4), 534-538 (2007).

Chowdhury, A. Historicizing, theorizing, and contextualizing feminism, ICFAI J. of English Studies, 4(1), 28-39 (2009).

Cubillas M, Abril E., y otros cuatro autores, Creencias sobre estereotipos de género de jóvenes universitarios del norte de México, Diversitas: Perspectivas en Psicología 12(2), 217-230 (2016).

Davids, T., Van Driel, F., y Parren, F. Feminist change revisited: gender mainstreaming as slow revolution. Journal of International Development, 26(3), 396-408 (2014).

Galicia-Alarcón, L., Balderrama-Trápaga, J., y Edel-Navarro R., Validez de contenido por juicio de expertos: propuesta de una herramienta virtual, http://dx.doi.org/10.32870/Ap.v9n2.993, Apertura, 9(2), 42-53 (2017).

Gambara, H., y Vargas T., Medición del grado de sensibilidad frente al enfoque basado en derechos humanos y perspectiva de género en intervenciones psicosociales, http://dx.doi.org/10.5093/in2012v21n1a8, Psychosocial Intervention 1(1), 3-15 (2012).

García, A., Género y desarrollo humano: una relación imprescindible. Agencia Española de Cooperación Internacional al Desarrollo (AECID), Madrid, (2009).

García, M. y Castro A., Análisis de los estereotipos de género en alumnado de formación profesional: diferencias según sexo, edad y grado, https://doi.org/10.6018/rie.35.1.257191, Investigación Educativa 35(1), 151-65 (2017).

Herrera-Seda, C., Pérez-Salas, C., y Echeita, G. Teorías implícitas y prácticas de enseñanza que promueven la inclusión educativa en la universidad. instrumentos y antecedentes para la reflexión y discusión, https://dx.doi.org/10.4067/S0718-50062016000500006, Formación Universitaria, 9 (5), 49-64 (2016).

Kwesiga, J. C., y Ssendiwala, E. N., Gender mainstreaming in the university context: prospects and challenges at makerere university, Uganda, https://doi.org/10.1016/j.wsif.2006.10.002, Women's studies international forum, 29(6), 592-605 (2006).

Larrondo, A., y Rivero, D., A case study on the incorporation of gender-awareness into the university journalism curriculum in Spain, https://doi.org/10.1080/09540253.2016.1270420, Gender and Education, 31(1), 1-14 (2017).

Lyle-Gonga M., A critical analysis of gender mainstreaming, https://doi.org/10.1017/S1743923X13000056, Politics \& Gender, 9(2), 209-213 (2013).

Maes, C., Schreurs, L., van Oosten, J., y Vandenbosch, L. \#(Me) too much? The role of sexualizing online media in adolescents' resistance towards the metoo-movement and acceptance of rape myths, https://doi.org/10.1016/j.adolescence.2019.10.005, Journal of adolescence, 77, 59-69 (2019).

Mamani, V., Herrera, D., y Arias, W. Análisis comparativo de machismo sexual en estudiantes universitarios peruanos y chilenos, https://dx.doi.org/10.4067/S0717-92272020000200106, Revista Chilena de Neuro-Psiquiatría, 58(2), 106-115 (2020).

Mayobre P., La formación de la identidad de género una mirada desde la filosofía. Revista Venezolana de Estudios de la Mujer, 12(28), 35-62 (2007).

Minto, R., y Mergaert, L., Gender mainstreaming and evaluation in the eu: comparative perspectives from feminist institutionalism, https://doi.org/10.1080/14616742.2018.1440181, International Feminist Journal of Politics, 20(2), 204220 (2018).

Moher, D., Shamseer, L., Clarke, M. y otros cinco autores, Preferred reporting items for systematic review and metaanalysis protocols (PRISMA-P) 2015 statement, https://doi.org/10.1186/2046-4053-4-1, Syst Rev 4,(1), (2015).

Montero, I., Aparicio D, y otros cinco autores, Género y salud mental en un mundo cambiante. Gac Sanit, 18 (1), 175 181 (2004).

Morley, L., The rules of the game: women and the leaderist turn in higher education, https://doi.org/10.1080/09540253.2012.740888, Gender and education, 25(1), 116-131 (2013).

NU, Naciones Unidas. Informe del consejo económico y social, año 1997, suplemento N 3 (A/52/3/Rev.1) (1999).

Ordorika, I., Equidad de género en la educación superior, Educación Superior, 44(174), 7-17 (2015).

Ramiro-Sánchez, T., Ramiro, M., Bermúdez, M., y Buela-Casal, G., Sexism in adolescent relationships: a systematic review, https://doi.org/10.5093/pi2018a19,. Psychosocial Intervention, 23(3), 123,132 (2018a).

Ramiro-Sánchez, T., Ramiro, M., Bermúdez, M., y Buela-Casal, G., Sexism and sexual risk behavior in adolescents: gender differences. International, https://doi.org/10.1016/j.ijchp.2018.04.002, Journal of Clinical and Health Psychology, 18(3), 245--253 (2018b).

Woolgar, M., y Scott, S., The negative consequences of over-diagnosing attachment disorders in adopted children: The importance of comprehensive formulations, https://doi.org/10.1177/1359104513478545, Clinical Child Psychology and Psychiatry, 19, 355-366 (2014). 